\title{
Análisis de la escolarización desde un punto de vista demográfico
}

Carmina Pérez Pérez

Universidad de Murcia

carpp@um.es

RESUMEN

Con este artículo nos proponemos, tomando como referentes el bagaje teórico e instrumental de la Demografía, dotar a la Demografía de la Educación de instrumentos válidos de análisis, a fin de crear su propio cuerpo de conocimientos, hasta ahora escasamente desarrollado.

Desde este objetivo se busca profundizar en el análisis de algunos aspectos de la escolarización mediante una serie de técnicas de medida que ponen de manifiesto rasgos sobresalientes de la población escolar. Concretamente, se desarrolla un instrumento base que hemos denominado Tabla de Indicadores Escolares (TIE), que se presenta como un instrumento de medida potente, capaz de aportar la información necesaria para responder al objetivo básico de este trabajo.

Palabras clave: Escolarización, Indicadores Escolares, Demografía de la Educación. 


\section{INTRODUCCIÓN}

La problemática y las aportaciones que se hacen en este artículo las consideramos como propias y fronterizas al campo de la Demografía de la Educación. Esta disciplina tiene como objetivo analizar el proceso de escolarización desde la perspectiva demográfica; por lo tanto, el examen de la escolarización, desde esta perspectiva, se hace recurriendo tanto al bagaje conceptual como instrumental que utiliza la Demografía, como disciplina matriz.

Como sucede en muchos ámbitos disciplinares, también en éste se utiliza no una única denominación para identificar este territorio científico, que en nuestro caso titulamos Demografía de la Educación. Las denominaciones que vienen usándose al respecto son próximas a ésta, tal como podemos ver a continuación.

Así, Paul Clerc utiliza la expresión Población Escolar (Clerc, 1977); por su parte, JeanLuc Guyot emplea en sus artículos los términos Démographie scolaire (Guyot, 1995, 1996), al igual que Melle Roseau Fabienne; en este caso, el autor estudia los efectivos de los alumnos de Primer y Segundo Grados (Roseau, 1998). También la expresión Démographie scolaire, y para los mismos niveles de estudio, es empleada en Recherche Bibliographique sur la Démographie Scolaire en France (1990-1998) (Viarouge, 19971998).

Otra expresión utilizada en el estudio de esta población es Demografía Educativa (Torres, 1991). Algunos autores se introducen a esta parcela del estudio de la demografía usando términos generales; así, aluden al estudio de la educación desde el punto de vista demográfico (Leguina, 1989). Demografía y Educación fue el nombre dado a unas Jornadas celebradas en el País Vasco en las que se trató de hacer un reconocimiento de algunos aspectos del Sistema Educativo desde la vertiente demográfica (Departamento de Educación, Universidades e Investigación, Gobierno Vasco, 1990).

Lo importante, a pesar de estas diferencias nominales, es la coincidencia básica en todas ellas en relación al objeto y cometido de este campo disciplinar. 


\section{LA TABLA DE INDICADORES ESCOLARES (TIE) COMO TÉCNICA DEMOGRÁFICA PARA EL ANÁLISIS DE LA ESCOLARIZACIÓN}

Este artículo aborda el estudio de la escolarización a través de una herramienta demográfica que llamamos Tabla de Indicadores Escolares (TIE). Partimos del hecho de que nuestro objeto de estudio es una población. Una población se sostiene como tal en tanto que conjunto de individuos que se identifican por alguna característica que todos ellos tienen en común. En nuestro caso la característica común, que define a la población objeto de estudio de la Demografía de la Educación, es formar parte del Sistema Educativo.

Tomando prestado el concepto de Demografía — ciencia que ha desarrollado todo un cuerpo de conocimientos importante-, centrado en «el estudio de aquellos procesos que determinan la formación, la conservación y la desaparición de las poblaciones» (Livi-Bacci, 1993), podemos advertir que, en un sentido amplio, también dentro del Sistema Educativo se dan procesos que determinan la formación (entradas en el Sistema Educativo), la conservación (permanencia en el Sistema Educativo) y desaparición (salidas del Sistema Educativo) de la población.

La definición clásica de demografía y sus herramientas deben ser referentes útiles para dotar a la Demografía de la Educación de instrumentos válidos de análisis y generar así su propio cuerpo de conocimientos, hasta ahora escasamente desarrollado. Nuestra intención y objetivo es trabajar en esta dirección, profundizando en algunos aspectos de la escolaridad, y lo vamos a hacer mediante una serie de técnicas de medida que ponen de manifiesto rasgos sobresalientes de la población escolar. Concretamente, el cuerpo de los datos estadísticos que vamos a utilizar va a estar apoyado en un instrumento base que consideramos oportuno llamarlo Tabla de Indicadores Escolares. En el artículo aludiremos a ella, de manera abreviada, con sus siglas: Tabla de Indicadores Escolares (TIE). Otra opción es hablar de Tabla de Vida Escolar, como ya hiciera Paul Clerc (Clerc, 1977), pero, al ofrecer nuestra tabla una descripción mayor de herramientas, es por lo que se ha tomado esa decisión. En la TIE, lo que la información estadística revela no es sólo la cuantificación de unos fenómenos, es también el reflejo de un Sistema Educativo y de unas políticas.

Por tanto, la finalidad principal que persigue este estudio es analizar la dinámica de la escolarización mediante la aplicación de dicha técnica demográfica (TIE), a fin de, como señala François Furet, "constituir el hecho histórico en series temporales de unidades homogéneas y comparable, y poder así medir su evolución por intervalos de tiempo dados» (Furet, 1978). 


\section{DESCRIPCIÓN DE LA TABLA DE INDICADORES ESCOLARES}

La tabla, que a continuación describimos -elaborada con los datos de la Región de Murcia del último Censo de Población y que se ha tomado como ejemplo para la explicación-, se presenta como un instrumento de medida y expresión de indicadores capaz de generar información útil para conocer comportamientos de interés de la población escolar. Comportamientos que permiten conocer el proceso de escolarización, el cual ocupa un lugar central tanto en el ámbito de la educación como en el social y cultural.

Nos apoyaremos, para conocer cómo funciona el Sistema Educativo, en una serie de indicadores (Tasa de escolarización, Esperanza de Vida Escolar, Edad Media de Entrada...) que nos permiten, a través de la cuantificación, hacer un estudio longitudinal.

Adelantamos que la tabla se apoya sobre la base de la hipótesis de la generación ficticia, convención usual.

La Tabla de Indicadores Escolares (TIE), en este caso de la Región de Murcia de 1991 (tabla 1), está formada por 13 columnas: Columnas de 1 a 11 y Columnas 6' y 8'. La razón que justifica esta extensión es la de ofrecer todos los pasos que van generando el desarrollo del cuadro. Es la manera de hacer visible todo el proceso que culmina con el indicador más importante que de ella se deriva, la Esperanza de Vida Escolar, si bien otros indicadores son también facilitados para una mejor comprensión del estudio de la escolaridad. 
TABLA 1 (A)

Tabla de Indicadores Escolares (TIE). Región de Murcia, 1991. Total

\begin{tabular}{|c|c|c|c|c|c|c|c|}
\hline$x$ & $\mathrm{E}_{\mathrm{x}}$ & $P_{x}$ & $S_{x}$ & $N\left(S_{x}\right)$ & $e_{(x, x+1)}$ & $\mathrm{xpe}_{\mathrm{x}}$ & $\mathrm{p}_{\mathrm{x}}$ \\
\hline Edad & Estudiantes & $\begin{array}{r}\text { Población } \\
\text { total }\end{array}$ & $\begin{array}{c}\text { \% tasa } \\
\text { escola- } \\
\text { rización }\end{array}$ & $\begin{array}{r}\% \text { tasa } \\
\text { no esco- } \\
\text { larizados }\end{array}$ & Entradas & $\begin{array}{r}\text { Edad } \\
\text { ponderada } \\
\text { entradas }\end{array}$ & $\begin{array}{r}\% \text { cociente } \\
\text { de } \\
\text { entrada }\end{array}$ \\
\hline 1 & 2 & 3 & 4 & 5 & 6 & $6^{\prime}$ & 7 \\
\hline 4 & 12.148 & 13.561 & 90 & 10 & 8 & 32 & 80 \\
\hline 5 & 13.727 & 14.078 & 98 & 2 & 1 & 5 & 50 \\
\hline 6 & 14.147 & 14.262 & 99 & 1 & 1 & 6 & 100 \\
\hline 7 & 14.954 & 15.023 & 100 & 0 & 0 & 0 & 0 \\
\hline 8 & 16.000 & 16.054 & 100 & 0 & 0 & 0 & 0 \\
\hline 9 & 16.800 & 16.845 & 100 & 0 & 0 & 0 & 0 \\
\hline 10 & 17.883 & 17.939 & 100 & 0 & 0 & 0 & 0 \\
\hline 11 & 18.449 & 18.555 & 99 & 1 & 0 & 0 & 0 \\
\hline 12 & 19.061 & 19.216 & 99 & 1 & 0 & 0 & 0 \\
\hline 13 & 18.518 & 18.780 & 99 & 1 & 0 & 0 & 0 \\
\hline 14 & 18.215 & 19.412 & 94 & 6 & 0 & 0 & 0 \\
\hline 15 & 16.227 & 19.603 & 83 & 17 & 0 & 0 & 0 \\
\hline 16 & 13.544 & 19.546 & 69 & 31 & 0 & 0 & 0 \\
\hline 17 & 11.729 & 19.453 & 60 & 40 & 0 & 0 & 0 \\
\hline 18 & 9.806 & 18.951 & 52 & 48 & 0 & 0 & 0 \\
\hline 19 & 8.032 & 18.584 & 43 & 57 & 0 & 0 & 0 \\
\hline 20 & 6.894 & 18.899 & 36 & 64 & 0 & 0 & 0 \\
\hline 21 & 5.736 & 18.616 & 31 & 69 & 0 & 0 & 0 \\
\hline 22 & 4.799 & 18.658 & 26 & 74 & 0 & 0 & 0 \\
\hline 23 & 3.870 & 18.876 & 21 & 79 & 0 & 0 & 0 \\
\hline 24 & 3.009 & 18.408 & 16 & 84 & 0 & 0 & 0 \\
\hline 25 & 2.220 & 17.996 & 12 & 88 & 0 & 0 & 0 \\
\hline 26 & 1.712 & 17.820 & 10 & 90 & 0 & 0 & 0 \\
\hline 27 & 1.268 & 17.400 & 7 & 93 & 0 & 0 & 0 \\
\hline 28 & 1.018 & 15.991 & 6 & 94 & 0 & 0 & 0 \\
\hline 29 & 796 & 15.508 & 5 & 95 & 0 & 0 & 0 \\
\hline \multirow[t]{2}{*}{$\geq 30$} & 6.000 & 533.640 & 1 & 99 & 0 & 0 & 0 \\
\hline & & & & & 10 & 43 & \\
\hline
\end{tabular}


TABLA 1 (B)

Tabla de Indicadores Escolares (TIE). Región de Murcia, 1991. Total

\begin{tabular}{|c|c|c|c|c|c|}
\hline$x$ & $S_{(x, x+1)}$ & $\mathrm{xps}_{\mathrm{x}}$ & $q_{x}$ & $\sum_{6}^{\geq 30} S_{x}$ & $e v_{x}$ \\
\hline Edad & Salidas & $\begin{array}{r}\text { Edad ponderada } \\
\text { salidas }\end{array}$ & $\begin{array}{r}\% \text { cociente } \\
\text { de salida }\end{array}$ & Total años & $\begin{array}{r}\text { Esperanza de } \\
\text { vida escolar }\end{array}$ \\
\hline 1 & 8 & $8^{\prime}$ & 9 & 10 & 11 \\
\hline 4 & 0 & 0 & 0 & - & - \\
\hline 5 & 0 & 0 & 0 & - & - \\
\hline 6 & 0 & 0 & 0 & 1.368 & 13,3 \\
\hline 7 & 0 & 0 & 0 & 1.269 & 12,2 \\
\hline 8 & 0 & 0 & 0 & 1.169 & 11,2 \\
\hline 9 & 0 & 0 & 0 & 1.069 & 10,2 \\
\hline 10 & 1 & 10 & 1 & 969 & 9,2 \\
\hline 11 & 0 & 0 & 0 & 869 & 7,6 \\
\hline 12 & 0 & 0 & 0 & 707 & 7,3 \\
\hline 13 & 5 & 65 & 5 & 671 & 6,3 \\
\hline 14 & 11 & 154 & 12 & 572 & 5,6 \\
\hline 15 & 14 & 210 & 17 & 478 & 5,3 \\
\hline 16 & 9 & 144 & 13 & 395 & 5,2 \\
\hline 17 & 8 & 136 & 13 & 326 & 4,9 \\
\hline 18 & 9 & 162 & 17 & 266 & 4,6 \\
\hline 19 & 7 & 133 & 16 & 214 & 4,5 \\
\hline 20 & 5 & 100 & 14 & 171 & 4,3 \\
\hline 21 & 5 & 105 & 16 & 135 & 3,9 \\
\hline 22 & 5 & 110 & 19 & 104 & 3,5 \\
\hline 23 & 5 & 115 & 24 & 78 & 3,2 \\
\hline 24 & 4 & 96 & 25 & 57 & 3,1 \\
\hline 25 & 2 & 50 & 17 & 41 & 2,9 \\
\hline 26 & 3 & 78 & 30 & 29 & 2,4 \\
\hline 27 & 1 & 27 & 14 & 19 & 2,2 \\
\hline 28 & 1 & 28 & 17 & 12 & 1,5 \\
\hline 29 & 4 & 116 & 80 & 6 & 0,7 \\
\hline \multirow[t]{3}{*}{$\geq 30$} & 1 & - & 100 & 1 & 0,5 \\
\hline & 99 & 1.839 & & & \\
\hline & $S_{x}-S_{x+1}$ & $\mathrm{~s}_{(\mathrm{x}, \mathrm{x}+1) \mathrm{x}}$ & $\left(S_{(x, x+1)} / S_{x}\right) 100$ & $\sum_{6}^{\geq 30} s_{x}$ & $1 / 2+\left(\Sigma\left(S_{x+1}\right) / S_{x}\right)$ \\
\hline${ }_{\bar{x}} e=4,3$ & & & $\bar{x} s=18,6$ & & $e v_{\bar{x}}=14,3$ \\
\hline
\end{tabular}

FUENTES:

Columnas 2 y 3: Centro Regional de Estadística de Murcia, Censo de Población de 1991 de la Región de Murcia. Resultados Regionales. Columnas 4 a 11: Elaboración propia. 
La tabla presenta, por otra parte, varios cuerpos en horizontal claramente diferenciados, concretamente ocho. El primero explicita el título; a través de él sabemos qué ofrece el cuadro y nos pone en conocimiento de qué población estamos estudiando. El segundo contiene las representaciones de cada una de las columnas; son, por otra parte, las variables base de las fórmulas. El tercero viene a ser una expresión explicativa del anterior. El cuarto no tiene otro valor que facilitar la identificación de cada columna para la explicación de la tabla. El quinto contiene la parte variable de la TIE, los datos. El sexto comunica cómo se han generado los datos. Proporciona la fórmula que permite el cálculo de cada una de las columnas. El séptimo (tabla (B)) ofrece tres indicadores escolares: la Edad media de entrada, la Edad media de salida y la Esperanza de vida media. Y en el octavo (tabla (B)) se especifican las fuentes de datos utilizadas. Vista, a grosso modo, la estructura de la TIE, pasemos a continuación a revisar cada una de sus columnas.

Columna 1. x (Edad). Contempla todas las edades de la población estudiante, tal y como es clasificada en el Censo de Población de 1991 de la Región de Murcia (Centro Regional de Estadística de Murcia, 1994). El último registro ( $\geq 30)$, al ser un dato agrupado, genera estadísticas, en la última fila, que serán útiles para mostrar ciertas informaciones y metodológicamente necesarias para realizar determinados cálculos, pero en algunos casos no tiene sentido la interpretación, son datos utilizados para el desarrollo de la tabla pero no siempre observados en nuestro análisis.

Columna 2. $\mathrm{E}_{\mathrm{x}}$ (Estudiantes). Población escolarizada por edad, tomada del Censo de Población de 1991 de la Región de Murcia.

Columna 3. $\mathrm{P}_{\mathrm{x}}$ (Población total). Población por edad, tomada igualmente del Censo de Población de 1991 de la Región de Murcia.

Estas primeras columnas contienen información directa del Censo, que son la base para generar nuevos datos o datos que podemos llamar derivados.

Columna 4. $\mathrm{S}_{\mathrm{x}}$ (Tasa de escolarización). Esta columna está formada por la proporción de la población general que es estudiante, expresada por edad y en tantos por ciento. Igualmente se podían haber presentado los datos en tantos por mil o por cualquier otra proporción; la razón que explica la decisión de expresar esta tasa en relación a una generación ficticia de 100 individuos es la de mostrar datos en unos términos con los que se suele estar más familiarizado.

$$
S_{x}=\left(E_{x} / P_{x}\right) 100
$$

Ej. $\mathrm{S}_{6}=\left(\mathrm{E}_{6} / \mathrm{P}_{6}\right) 100$

$S_{6}=(14.147 / 14.262) 100$

$\mathrm{S}_{6}=99$ 
Interpretación. De cada 100 personas de la población general de 6 años hay escolarizadas 99.

Columna 5. $\mathrm{N}\left(\mathrm{S}_{\mathrm{x}}\right)$ (Tasa de no escolarizados). En este caso son los otros, la proporción de los individuos de la población general por edad que no forman parte del Sistema Educativo. En tanto que complemento de la anterior, su cálculo es fruto de restarle a la generación de los 100 individuos de la población general el número de los escolarizados, esto es:

$$
N\left(S_{x}\right)=100-S_{x}
$$

Ej. $\mathrm{N}\left(\mathrm{S}_{6}\right)=100-\mathrm{S}_{6}$

$N\left(S_{6}\right)=100-99$

$N\left(S_{6}\right)=1$

Interpretación. De cada 100 personas de la población general de 6 años hay una que no está escolarizada.

Columna 6. $e_{(x, x+1)}$ (Entradas). Son los individuos que estando escolarizados en la edad ${ }_{x+1}$ no lo estaban en la edad ${ }_{x}$. Son, por tanto, nuevas entradas al Sistema Educativo. Si a la edad ${ }_{x+1}$ le resto los estudiantes que ya había en la edad anterior (edad ${ }_{x}$ ), obtengo los que se incorporan por primera vez al Sistema Educativo. Cuando ya no se producen entradas sino el fenómeno contrario, salidas, el resultado de la resta es un valor negativo, en cuyo caso, al carecer de sentido a los efectos que aquí pretendemos, se sustituye por el valor cero.

Esta columna nos informa de cómo los 100 individuos de la generación que estamos estudiando se incorporan gradualmente en las distintas edades. La fórmula que nos facilita este dato es, por tanto:

$$
e_{(x, x+1)}=S_{x+1}-S_{x}
$$
Ej. $\mathrm{e}_{(6,6+1)}=\mathrm{S}_{6+1}-\mathrm{S}_{6}$
$\mathrm{e}_{(6,7)}=\mathrm{S}_{7}-\mathrm{S}_{6}$
$\mathrm{e}_{(6,7)}=100-99$
$e_{(6,7)}=1$

Interpretación. De 7 años hay sólo un niño por cada 100 que, estando fuera del Sistema Educativo, se incorpora a formar parte del grupo de niños escolarizados. El resto, el 99\% de los niños de 7 años, ya lo estaban. Esto es, en $S_{7}=100$ sólo uno es nueva entrada.

Columna 7. $\mathrm{p}_{\mathrm{x}}$ (Cociente de entrada). Es la proporción de la población que entra nueva a formar parte del Sistema Educativo en relación a los no escolarizados por edad, expresada en tantos por ciento. Su cálculo sería el siguiente:

$$
p_{x}=\left(e_{(x, x+1)} / N\left(S_{x}\right)\right) 100
$$


Ej. $\mathrm{p}_{6}=\left(\mathrm{e}_{(6,6+1)} / \mathrm{N}\left(\mathrm{S}_{6}\right)\right) 100$

$$
\begin{aligned}
& \mathrm{p}_{6}=\left(\mathrm{e}_{(6,7)} / \mathrm{N}\left(\mathrm{S}_{6}\right)\right) 100 \\
& \mathrm{p}_{6}=100
\end{aligned}
$$$$
p_{6}=(1 / 1) 100
$$

Interpretación. A la edad de 6 años el cien por cien de los niños no escolarizados pasan a estarlo, esto es, pasan a formar parte del Sistema Educativo.

Columna 8. $\mathrm{s}_{(\mathrm{x}, \mathrm{x}+1)}$ (Salidas). Si en las Columnas 6 y 7 operábamos con las entradas, ahora en las Columnas 8 y 9 operaremos con las salidas del Sistema Educativo. Desde la misma lógica, la Columna 8 contempla, en esta ocasión, los individuos que estando escolarizados a una edad ${ }_{x}$ dejan de estarlo a la edad ${ }_{x+1}$. Es un valor que nos informa de cómo los 100 individuos de la generación que estamos estudiando salen gradualmente del Sistema Educativo. Como es obvio, la suma de todas las salidas es 100. Tomamos como base para el cálculo los alumnos escolarizados; aquí nos interesa observar las edades en las que los valores, fruto de las salidas, disminuyen. El contenido de esta columna se obtiene cambiando el orden de los factores de la recta que operaban en las entradas, e igualmente sustituyendo los valores negativos por 0 . La fórmula es ahora:

$$
S_{(x, x+1)}=S_{x}-S_{x+1}
$$

$$
\begin{array}{cl}
E j . \mathrm{s}_{(18,18+1)}=\mathrm{S}_{18}-\mathrm{S}_{18+1} & \mathrm{~s}_{(18,19)}=\mathrm{S}_{18}-\mathrm{S}_{19} \quad \mathrm{~s}_{(18,19)}=52-43 \\
\mathrm{~s}_{(18,19)}=9
\end{array}
$$

Interpretación. De la población que estamos contemplando, formada por 100 escolarizados, salen del Sistema Educativo a la edad de 18 años 9 de ellos.

Columna 9. $\mathrm{q}_{\mathrm{x}}$ (Cociente de salida). Es la proporción de la población escolarizada que deja de estarlo, que abandona el Sistema Educativo. El cociente de salida viene dado en tantos por ciento y su fórmula viene expresada como sigue:

$$
\begin{gathered}
\mathrm{q}_{\mathrm{x}}=\left(\mathrm{s}_{(\mathrm{x}, \mathrm{x}+1)} / \mathrm{s}_{\mathrm{x}}\right) 100 \\
\mathrm{q}_{18}=\left(\mathrm{s}_{(18,19)} / \mathrm{s}_{18}\right) 100 \quad \mathrm{q}_{18}=(9 / 52) 100 \\
\mathrm{q}_{18}=17
\end{gathered}
$$

Interpretación. A la edad de 18 años el 17\% de la población escolarizada abandona el Sistema Educativo. 
Columna 10. $\Sigma_{6}^{\geq 30} \mathrm{~S}_{\mathrm{x}}$ (Total años). Los datos de esta columna están formados por las Tasas de escolarización $\left(S_{x}\right)$ acumuladas. El superíndice $\left({ }^{30}\right)$ y subíndice $\left.{ }_{6}\right)$ del sumatorio expresan el orden de la suma, esto es, el cálculo se inicia desde la última edad ( $\geq 30$ ), hasta la edad de 6 años. No se han tenido en cuenta en este cálculo las edades de escolaridad no obligatoria, edades 4 y 5 . El hecho de ofrecer datos a partir de los 6 años permite facilitar información de mayor interés, teniendo presente que el sentido de esta columna es, a partir de ella, hallar la Esperanza de Vida Escolar (Columna 11). La expresión de la cabecera de este párrafo es la que representa adecuadamente la manera de operar, esto es:

Ej. $\sum_{6}^{\geq 30} \mathrm{~S}_{25}=41$

$$
\sum_{6}^{\geq 30} \mathrm{~S}_{\mathrm{x}}
$$

Interpretación. Los 12 individuos escolarizados a la edad de 25 años esperan permanecer, entre todos ellos, 41 años en el Sistema Educativo.

Columna 11. ev ${ }_{\mathrm{x}}$ (Esperanza de Vida Escolar). Éste es uno de los indicadores más importantes en el estudio de la vida escolar de una población; es, por decirlo de alguna manera, el homólogo de la Esperanza de Vida de las tablas de mortalidad. La Esperanza de Vida Escolar es un indicador que resume bien la experiencia escolar de una población; de ahí que su uso sea bastante idóneo para el estudio evolutivo del comportamiento de la escolarización a lo largo de un período de tiempo e, igualmente, para establecer comparaciones entre distintas poblaciones. La Esperanza de Vida Escolar $\left(\mathrm{ev}_{\mathrm{x}}\right)$ nos indica lo que espera cada individuo permanecer en el Sistema Educativo según su edad. En tanto que la $e v_{x}$ es un valor individualizado, su cálculo es el resultado de dividir los años de vida escolar acumulados, del grupo de individuos escolarizados de una edad determinada (Columna 10), entre los individuos que forman el grupo de esa edad (Columna 4), para poder concluir que un estudiante de una edad determinada espera permanecer en el Sistema Educativo un número de años equis. Este cálculo se concreta sumándole al cociente $1 / 2$; esto corrige el dato, en el sentido de aproximarlo a un valor medio anual más preciso. Su fórmula sería, por tanto:

$$
e v_{x}=1 / 2+\left(\Sigma\left(S_{x+1}\right) / S_{x}\right)
$$

$E j . \mathrm{ev}_{19}=1 / 2+\left(\Sigma\left(\mathrm{S}_{19+1}\right) / \mathrm{S}_{19}\right) \quad \mathrm{ev} \mathrm{v}_{19}=1 / 2+\left(\Sigma\left(\mathrm{S}_{20}\right) / \mathrm{S}_{19}\right) \quad \mathrm{ev} \mathrm{v}_{19}=1 / 2+(171 / 43) \quad \mathrm{ev}_{19}=4,5$ Interpretación. Un estudiante de 19 años espera permanecer en el Sistema Educativo 4,5 años.

Las columnas que a continuación vamos a describir (6' y 8') nos permiten hallar dos indicadores: Edad media de entrada al Sistema Educativo y Edad media de salida del Sistema 
Educativo, y deducir un tercero, la Esperanza de vida escolar media o Duración media de la escolaridad.

Columna $6^{\prime} \cdot \overline{\mathrm{x}}$ e (Edad media $(\overline{\mathrm{x}})$ de entrada (e)). Se trata en esta columna de multiplicar las entradas por la edad, esto es, $e_{(x, x+1)}$, con el fin de darle a cada una de ellas su peso específico en función de la edad que le corresponde. La suma de esta columna dividida por la suma de todas las entradas nos da el valor medio de entrada al Sistema Educativo de la población de estudiantes que estamos analizando. El cálculo de la $\overline{\bar{x}}_{\overline{\mathrm{x}}}$ e se obtiene dividiendo el sumatorio de la edad ponderada de las entradas $\left(\Sigma x p e_{x}\right)$ entre el sumatorio de las entradas $\left(\Sigma e_{(x, x+1)}\right)$, esto es:

$$
\bar{x} e=\Sigma x_{x} / \Sigma e_{(x, x+1)}
$$

Región de Murcia, 1991. Total

$$
\bar{x} e=43 / 10 \quad \bar{x} e=4,3
$$

Interpretación. La Edad media de entrada al Sistema Educativo de la población de estudiantes objeto de estudio es de 4,3 años de edad.

Columna $8^{\prime} \cdot \overline{\mathrm{x}} \mathrm{s}$ (Edad media $(\overline{\mathrm{x}})$ de salida $(\mathrm{s})$ ). Esta columna es una réplica de la columna anterior (6'), en esta ocasión en relación a las salidas. La edad media de salida se obtiene multiplicando las salidas por la edad, esto es, $s_{(x, x+1)}$, con el fin de darle a cada una de ellas su peso específico en función de la edad que le corresponde. La suma de esta columna, sin incluir el grupo de edad $\geq 30$, dividida por la suma de todas las salidas, excluido también el grupo de edad $\geq 30$, nos da el valor medio de salida del Sistema Educativo de la población de estudiantes que estamos analizando. Por razones obvias, en ambas sumas, se ha excluido el grupo de edad $\geq 30$, pues al no estar las edades desagregadas no es posible calcular el peso que le corresponde a cada una de ellas. Aquí el dividendo es el sumatorio de la edad ponderada de las salidas $\left(\Sigma x_{x p}\right)$ y el divisor el sumatorio de las salidas $\left(\Sigma \mathrm{s}_{(\mathrm{x}, \mathrm{x}+1)}\right)$. Lo que acabamos de decir puede expresarse del modo siguiente:

$$
\overline{\mathrm{x}} \mathrm{s}=\Sigma \mathrm{xps}_{\mathrm{x}} / \Sigma \mathrm{s}_{(\mathrm{x}, \mathrm{x}+1)}
$$

Región de Murcia, 1991. Total

$$
\overline{\mathrm{x}} \mathrm{S}=1.839 / 99 \quad \overline{\mathrm{x}} \mathrm{S}=18,6
$$

Interpretación. La Edad media de salida del Sistema Educativo de la población de estudiantes objeto de estudio es de 18,6 años. 
Esperanza de vida media. $\mathrm{ev}_{\overline{\mathrm{x}}}$. Para obtener la Esperanza de vida escolar media o Duración media de la escolaridad basta con restarle a la Edad media de salida $(\overline{\bar{x}} \mathrm{~s})$ la Edad media de entrada $\left({ }_{\bar{x}}\right.$ e $)$. Esto es:

$$
e v_{\bar{x}}=\frac{\bar{x}}{s}-{ }_{\bar{x}} e
$$

Región de Murcia, 1991. Total

$$
e v_{\bar{x}}=18,6-4,3 \quad e v_{\bar{x}}=14,3
$$

Interpretación. En el año 1991 los estudiantes de la Región de Murcia permanecían en el Sistema Educativo una media de 14,3 años.

Los indicadores Edad media de entrada $\left(\bar{x}_{\bar{x}}\right.$ e), Edad media de salida $\left({ }_{\bar{x}} s\right)$ y Esperanza de vida media $\left(\mathrm{ev}_{\overline{\mathrm{x}}}\right)$ están expresados en la base de la tabla.

\section{ESTUDIO DE LA ESCOLARIZACIÓN EN ESPAÑA}

Apoyándonos en la Tabla de Indicadores Escolares vamos a hacer algunas reflexiones sobre la dinámica de la escolarización en España —-tomando como años de referencia 1970, 1981 y 1991-. La elección de los años observados está condicionada por las estadísticas nacionales disponibles. La escolarización en España, en sus inicios, ha sido estudiada por los autores Jean-Louis Guereña y Antonio Viñao Frago (Guereña y Viñao, 1996); estos autores analizan el proceso de escolarización en España desde 1750 a 1850. Durante la segunda mitad del siglo XIX hay más estudios realizados al respecto, si bien son estudios sobre todo de ámbito regional o provincial (Ramos, 1987; Fernández, 1987; Lozano, 1990, etc.).

La fuente de información que vamos a manejar para la población general según edad será siempre la de los censos, y en relación a la población de estudiantes se utilizarán, para los años 1970 y 1981, los datos que facilita el INE en Estadística de la Enseñanza en España; y para el año 1991 dos fuentes: una para los estudiantes de 4 a 19 años, información obtenida de la Estadística de la Enseñanza en España que facilita el Ministerio de Educación y Ciencia, y otra para la edad de 20 y más años, en este caso el dato se ha extraído del Censo de Población de 1991. Resultados Nacionales, ya que la estadística del MEC sólo contiene las enseñanzas no universitarias.

Dos explicaciones en relación a la tabla de 1991: una, el hecho de que no se hayan tomado en su totalidad los datos del censo, en relación a la población de estudiantes, responde 
a que en esta fuente estadística los datos no vienen diferenciados edad a edad, cuestión importante para el tipo de análisis que deseamos desarrollar. Y dos, para el año 1991 no se ha podido continuar con la misma fuente de datos sobre estudiantes que para los dos años anteriores (1970 y 1981) porque: «El Ministerio de Educación y Ciencia asume, de acuerdo con el Instituto Nacional de Estadística, a partir de las correspondientes al curso escolar 1985-86, las funciones de recogida, procesamiento y publicación de resultados de las estadísticas de los niveles y modalidades de enseñanza no universitaria: Preescolar, General Básica, Bachillerato y Curso de Orientación Universitaria, Formación Profesional, Educación Permanente de Adultos, Educación Especial y otras enseñanzas» (INE, 1988). Interrumpiéndose así la publicación Estadísticas de la Enseñanza en España tal y como venía realizándose. Esta estadística del INE se limita, a partir del curso 1985-86, a ofrecer la información detallada de la Educación de Tercer Grado (Grado Superior), que comprende fundamentalmente la Educación Universitaria.

Si se examinan las Tablas de Indicadores Escolares de España para 1970, 1981 y 1991 y se observa la Columna 2 de las tablas 2, 3 y 5 (Estudiantes $\left(E_{x}\right)$ ), se aprecian algunas incoherencias respecto a lo que debería ser la dinámica normal en la curva de estudiantes por edad, y que se pueden justificar en cierta medida. Por ejemplo, el que a la edad de 6 años hayan más niños escolarizados que población le corresponde a dicha edad se explica, según el propio INE, porque un porcentaje de niños que no han cumplido 6 años han sido admitidos en Centros de EGB — sobre todo en núcleos de población donde no existen unidades de Educación Preescolar-y han sido computados como de 6 años de edad. EI INE señala también que el hecho de que aumente a los 20 años la población de estudiantes, cuando la tendencia es a disminuir progresivamente, se debe a que los estudiantes de determinadas enseñanzas con edades de más de 20 años han sido registrados en la categoría de edad de 20 y más. De manera singular, en la TIE de 1981 llama la atención el alto número de estudiantes en relación a la población de 6 a 12 años; el INE, en sus estadísticas, publica también datos de estudiantes para 1981 con la misma anomalía; éste argumenta que el hecho de que los estudiantes superen el tamaño de la población en dichas edades podría atribuirse a eventuales duplicaciones en el cómputo de la matrícula escolar. Nosotros pensamos que los beneficios económicos y ayudas que se obtienen en razón del número de alumnos pueden también justificar que las estadísticas que facilitan los colegios al MEC estén sobrevaloradas, lo cual puede explicar la desviación de dichos datos.

Estas razones explican algunos de los datos llamativos que aparecen en los registros de dichas edades. Para que las anomalías señaladas no afecten a los indicadores de la tabla, y poder mostrar la coherencia interna de las estadísticas, al mismo tiempo que aproximarnos en lo posible a la situación real de la escolarización de los años que se analizan, se 
han hecho las correcciones oportunas teniendo en cuenta la tendencia lógica de los datos. En cualquier caso, estas anomalías no hubiesen restado interés a las conclusiones, ya que si bien es cierto que se ven afectadas por ellas, lo hacen de manera irrelevante desde el punto de vista estadístico.

Las comparaciones entre las tablas de 1970 y 1981 son posibles, ya que los sesgos que acabamos de señalar, teóricamente, actúan de idéntica forma en ambos casos, partiendo, como así lo hacemos, de las mismas fuentes de información. Con la tabla de 1991 se dan dos peculiaridades que sólo permiten una comparación parcial con las anteriores. Por una parte, las fuentes de información no son las mismas y, por otra parte, el que se vayan produciendo progresivamente, a partir de 1978 con la estructura del Estado de las Autonomías, las transferencias de educación a las distintas regiones, hasta configurar el llamado «Territorio MEC», hacen que las series estadísticas dejen de estar centralizadas, que se pierda la serie de los datos, la uniformidad de los mismos e incluso el no registro de parte de ellos. Un ejemplo de irregularidad es el siguiente: el MEC facilita, en su publicación Estadísticas de la Enseñanza en España, el dato de 494.863 alumnos escolarizados, dato que no incluye en su serie de datos a efectos de cómputo para la elaboración de las tasas de escolarización, nosotros tampoco lo hacemos; no es posible incluirlo ya que estos 494.863 aparecen bajo la rúbrica de «No consta» (la edad); es, por tanto, un número importante de casos perdidos. Concluyendo, España en 1991 dispone ya de un escenario de transferencias educativas singulares que, al agrupar los datos que se extraen de las distintas Comunidades Autónomas para ofrecer datos nacionales, por las razones que hemos apuntado, generan ciertas diferencias respecto al pasado, que impiden la comparación al cien por cien con las tablas anteriores.

Para facilitar el análisis de las tablas se ha utilizado la misma serie de edades en todas ellas (de 4 a $\geq 20$ ). Una observación respecto a las edades: dada la no obligatoriedad y las características peculiares de la escolarización de los niños de 4 y 5 años, que lleva a situaciones singulares en las estadísticas, y dado que realmente empieza la escolaridad, desde 1970 con la LGE, a los 6 años, será, como ya se indicó anteriormente, a partir de esta edad desde la que generaremos la Esperanza de Vida Escolar $\left(\mathrm{ev}_{\mathrm{x}}\right)$, Columna 11; ello nos permitirá hacer un análisis comparativo más exacto y riguroso. Si bien la información correspondiente a las edades de 4 y 5 años es de interés para determinados componentes de la tabla y, especialmente, para el cálculo del indicador Edad media de entrada $\left(\bar{x}_{\bar{x}} \mathrm{e}\right)$.

Hechas estas consideraciones pasemos ahora a hacer algunas reflexiones sobre la dinámica de la escolarización desde 1970 hasta 1991 (tablas 2, 3 y 4). 
TABLA 2 (A)

Tabla de Indicadores Escolares (TIE). Total nacional, 1970

\begin{tabular}{|c|c|c|c|c|c|c|c|}
\hline $\mathrm{x}$ & $E_{x}$ & $P_{x}$ & $S_{x}$ & $N\left(S_{x}\right)$ & $e_{(x, x+1)}$ & $\mathrm{xpe}_{\mathrm{x}}$ & $p_{x}$ \\
\hline Edad & Estudiantes & $\begin{array}{r}\text { Población } \\
\text { total }\end{array}$ & $\begin{array}{c}\text { \% tasa } \\
\text { escola- } \\
\text { rización }\end{array}$ & $\begin{array}{r}\% \text { tasa } \\
\text { no esco- } \\
\text { larizados }\end{array}$ & Entradas & $\begin{array}{r}\text { Edad } \\
\text { ponderada } \\
\text { entradas }\end{array}$ & $\begin{array}{r}\% \text { cociente } \\
\text { de } \\
\text { entrada }\end{array}$ \\
\hline 1 & 2 & 3 & 4 & 5 & 6 & $6{ }^{\prime}$ & 7 \\
\hline 4 & 276.935 & 646.334 & 43 & 57 & 26 & 104 & 46 \\
\hline 5 & 449.839 & 656.254 & 69 & 31 & 32 & 160 & 103 \\
\hline 6 & 675.805 & 670.335 & 98 & 2 & 0 & 0 & 0 \\
\hline 7 & 626.614 & 645.158 & 98 & 2 & 0 & 0 & 0 \\
\hline 8 & 618.677 & 630.531 & 98 & 2 & 0 & 0 & 0 \\
\hline 9 & 607.235 & 617.674 & 98 & 2 & 1 & 9 & 50 \\
\hline 10 & 630.441 & 639.306 & 99 & 1 & 0 & 0 & 0 \\
\hline 11 & 599.764 & 614.355 & 98 & 2 & 0 & 0 & 0 \\
\hline 12 & 568.515 & 612.387 & 93 & 7 & 0 & 0 & 0 \\
\hline 13 & 511.867 & 599.767 & 85 & 15 & 0 & 0 & 0 \\
\hline 14 & 284.878 & 564.380 & 50 & 50 & 0 & 0 & 0 \\
\hline 15 & 196.994 & 560.989 & 35 & 65 & 0 & 0 & 0 \\
\hline 16 & 156.929 & 539.238 & 29 & 71 & 0 & 0 & 0 \\
\hline 17 & 121.578 & 545.163 & 22 & 78 & 0 & 0 & 0 \\
\hline 18 & 102.914 & 552.745 & 21 & 79 & 0 & 0 & 0 \\
\hline 19 & 83.630 & 511.201 & 20 & 80 & 0 & 0 & 0 \\
\hline \multirow[t]{2}{*}{$\geq 20$} & 323.588 & 21.871 .681 & 2 & 98 & 0 & 0 & 0 \\
\hline & & & & & 59 & 273 & \\
\hline
\end{tabular}


TABLA 2 (B)

Tabla de Indicadores Escolares (TIE). Total nacional, 1970

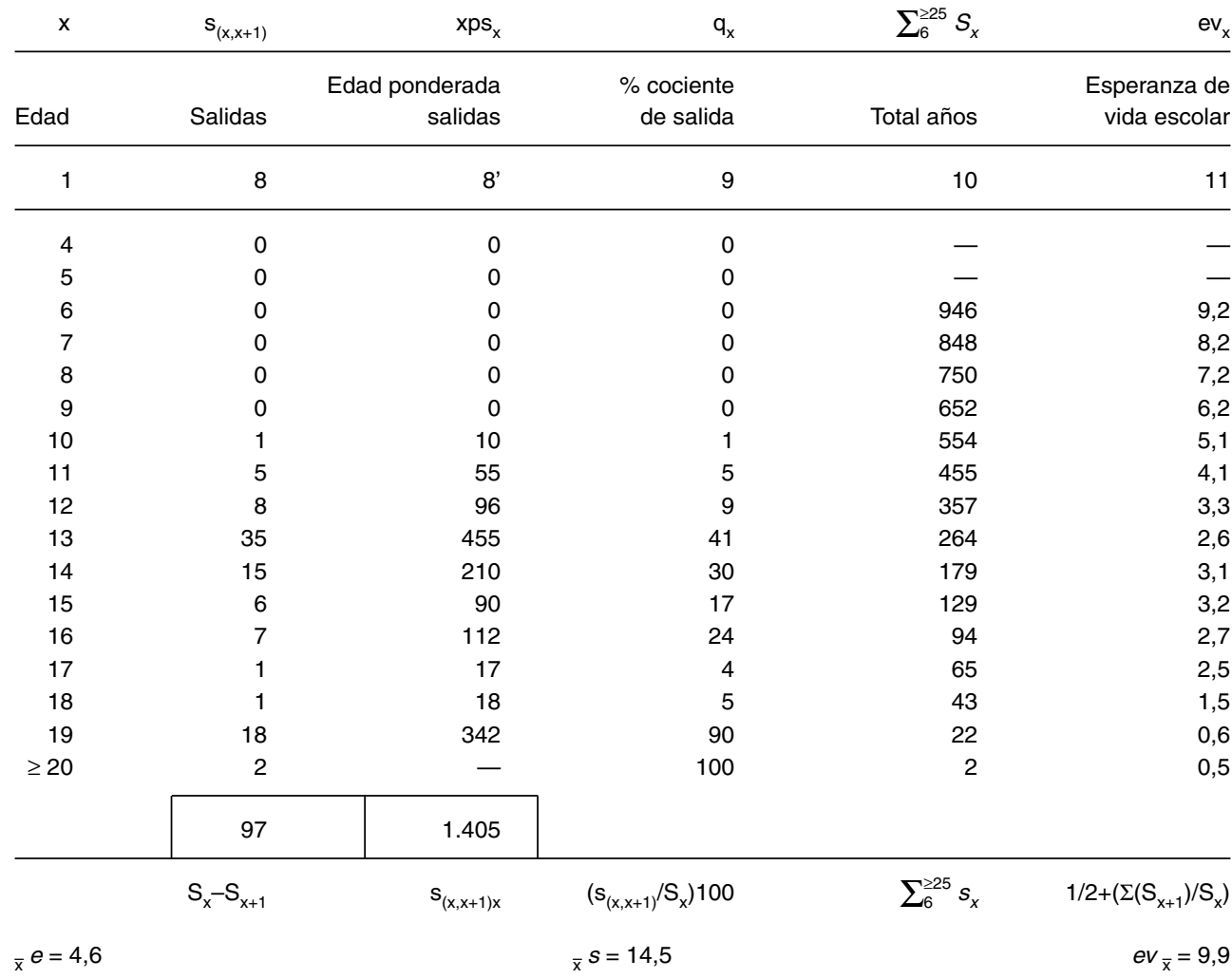

FUENTES:

Columna 2: INE, Estadística de Enseñanza en España (E.E.E.). Columna 3: INE, Censo de Población de España según la inscripción realizada al 31 de diciembre de 1970. Total Nacional. Columnas 4 a 11: Elaboración propia. 
TABLA $3(A)$

Tabla de Indicadores Escolares (TIE). Total nacional, 1981

\begin{tabular}{|c|c|c|c|c|c|c|c|}
\hline $\mathrm{x}$ & $E_{x}$ & $P_{x}$ & $S_{x}$ & $N\left(S_{x}\right)$ & $e_{(x, x+1)}$ & $\mathrm{xpe}_{\mathrm{x}}$ & $p_{x}$ \\
\hline Edad & Estudiantes & $\begin{array}{r}\text { Población } \\
\text { total }\end{array}$ & $\begin{array}{c}\text { \% tasa } \\
\text { escola- } \\
\text { rización }\end{array}$ & $\begin{array}{r}\% \text { tasa } \\
\text { no esco- } \\
\text { larizados }\end{array}$ & Entradas & $\begin{array}{r}\text { Edad } \\
\text { ponderada } \\
\text { entradas }\end{array}$ & $\begin{array}{r}\% \text { cociente } \\
\text { de } \\
\text { entrada }\end{array}$ \\
\hline 1 & 2 & 3 & 4 & 5 & 6 & $6{ }^{\prime}$ & 7 \\
\hline 4 & 455.204 & 653.623 & 70 & 30 & 23 & 96 & 80 \\
\hline 5 & 620.928 & 663.827 & 93 & 7 & 5 & 50 & 167 \\
\hline 6 & 690.846 & 666.831 & 98 & 2 & 1 & 6 & 0 \\
\hline 7 & 690.531 & 655.705 & 99 & 1 & 0 & 0 & 0 \\
\hline 8 & 688.803 & 663.359 & 98 & 2 & 1 & 8 & 0 \\
\hline 9 & 690.923 & 658.327 & 99 & 1 & 0 & 0 & 0 \\
\hline 10 & 681.853 & 655.462 & 98 & 2 & 0 & 0 & 0 \\
\hline 11 & 678.329 & 654.751 & 98 & 2 & 0 & 0 & 0 \\
\hline 12 & 658.233 & 645.881 & 97 & 3 & 0 & 0 & 0 \\
\hline 13 & 654.202 & 657.034 & 97 & 3 & 0 & 0 & 0 \\
\hline 14 & 577.029 & 689.200 & 84 & 16 & 0 & 0 & 0 \\
\hline 15 & 473.589 & 664.751 & 71 & 29 & 0 & 0 & 0 \\
\hline 16 & 369.143 & 680.342 & 54 & 46 & 0 & 0 & 0 \\
\hline 17 & 337.781 & 660.998 & 51 & 49 & 0 & 0 & 0 \\
\hline 18 & 236.198 & 635.029 & 37 & 63 & 0 & 0 & 0 \\
\hline 19 & 176.845 & 622.192 & 32 & 68 & 0 & 0 & 0 \\
\hline \multirow[t]{2}{*}{$\geq 20$} & 954.399 & 29.077 .902 & 3 & 97 & 0 & 0 & 0 \\
\hline & & & & & 30 & 160 & \\
\hline
\end{tabular}


TABLA $3(B)$

Tabla de Indicadores Escolares (TIE). Total nacional, 1981

\begin{tabular}{|c|c|c|c|c|c|}
\hline $\mathrm{x}$ & $S_{(x, x+1)}$ & $\mathrm{xps}_{\mathrm{x}}$ & $q_{x}$ & $\sum_{6}^{\geq 25} S_{x}$ & $e v_{x}$ \\
\hline Edad & Salidas & $\begin{array}{r}\text { Edad ponderada } \\
\text { salidas }\end{array}$ & $\begin{array}{r}\% \text { cociente } \\
\text { de salida }\end{array}$ & Total años & $\begin{array}{r}\text { Esperanza de } \\
\text { vida escolar }\end{array}$ \\
\hline 1 & 8 & 8' & 9 & 10 & 11 \\
\hline 4 & 0 & 0 & 0 & - & - \\
\hline 5 & 0 & 0 & 0 & - & - \\
\hline 6 & 0 & 0 & 0 & 1.116 & 10,9 \\
\hline 7 & 1 & 7 & 1 & 1.018 & 9,8 \\
\hline 8 & 0 & 0 & 0 & 919 & 8,8 \\
\hline 9 & 1 & 9 & 1 & 821 & 7,8 \\
\hline 10 & 0 & 0 & 0 & 722 & 6,9 \\
\hline 11 & 1 & 11 & 1 & 624 & 5,9 \\
\hline 12 & 1 & 12 & 1 & 526 & 4,9 \\
\hline 13 & 16 & 208 & 16 & 429 & 3,9 \\
\hline 14 & 13 & 182 & 15 & 332 & 3,5 \\
\hline 15 & 17 & 255 & 24 & 248 & 3,0 \\
\hline 16 & 3 & 48 & 6 & 177 & 2,8 \\
\hline 17 & 14 & 238 & 27 & 123 & 1,9 \\
\hline 18 & 5 & 162 & 13 & 72 & 1,4 \\
\hline 19 & 29 & 551 & 91 & 35 & 0,6 \\
\hline \multirow[t]{3}{*}{$\geq 20$} & 3 & - & 100 & 3 & 0,5 \\
\hline & 101 & 1.683 & & & \\
\hline & $S_{x}-S_{x+1}$ & $s_{(x, x+1) x}$ & $\left(\mathrm{~s}_{(\mathrm{x}, \mathrm{x}+1)} / \mathrm{S}_{\mathrm{x}}\right) 100$ & $\sum_{6}^{\geq 25} s_{x}$ & $1 / 2+\left(\Sigma\left(\mathrm{S}_{\mathrm{x}+1}\right) / \mathrm{S}_{\mathrm{x}}\right)$ \\
\hline$\overline{\mathrm{x}} e=5,3$ & & & $\overline{\mathrm{x}} S=16,7$ & & $e v_{\bar{x}}=11,4$ \\
\hline
\end{tabular}

FUENTES:

Columna 2: INE, Estadística de Enseñanza en España (E.E.E.). Columna 3: INE, Censo de Población de 1981. Resultados Nacionales. Columnas 4 a 11: Elaboración propia. 
TABLA 4 (A)

Tabla de Indicadores Escolares (TIE). Total nacional, 1991

\begin{tabular}{|c|c|c|c|c|c|c|c|}
\hline $\mathrm{x}$ & $E_{x}$ & $P_{x}$ & $S_{x}$ & $N\left(S_{x}\right)$ & $e_{(x, x+1)}$ & $\mathrm{xpe}_{\mathrm{x}}$ & $p_{x}$ \\
\hline Edad & Estudiantes & $\begin{array}{r}\text { Población } \\
\text { total }\end{array}$ & $\begin{array}{l}\text { \% tasa } \\
\text { escola- } \\
\text { rización }\end{array}$ & $\begin{array}{r}\% \text { tasa } \\
\text { no esco- } \\
\text { larizados }\end{array}$ & Entradas & $\begin{array}{r}\text { Edad } \\
\text { ponderada } \\
\text { entradas }\end{array}$ & $\begin{array}{r}\% \text { cociente } \\
\text { de } \\
\text { entrada }\end{array}$ \\
\hline 1 & 2 & 3 & 4 & 5 & 6 & $6{ }^{\prime}$ & 7 \\
\hline 4 & 398.780 & 421.654 & 95 & 5 & 4 & 16 & 80 \\
\hline 5 & 435.139 & 437.547 & 99 & 1 & 0 & 0 & 0 \\
\hline 6 & 463.342 & 471.546 & 98 & 2 & 2 & 12 & 100 \\
\hline 7 & 483.353 & 481.621 & 100 & 0 & 0 & 0 & 0 \\
\hline 8 & 501.419 & 513.017 & 98 & 2 & 1 & 8 & 50 \\
\hline 9 & 527.779 & 533.209 & 99 & 1 & 1 & 9 & 100 \\
\hline 10 & 558.381 & 560.226 & 100 & 0 & 0 & 0 & 0 \\
\hline 11 & 589.719 & 595.407 & 99 & 1 & 0 & 0 & 0 \\
\hline 12 & 611.476 & 625.866 & 98 & 2 & 0 & 0 & 0 \\
\hline 13 & 613.300 & 638.397 & 96 & 4 & 0 & 0 & 0 \\
\hline 14 & 636.914 & 665.906 & 96 & 4 & 0 & 0 & 0 \\
\hline 15 & 592.357 & 674.693 & 88 & 12 & 0 & 0 & 0 \\
\hline 16 & 494.275 & 672.797 & 73 & 27 & 0 & 0 & 0 \\
\hline 17 & 440.991 & 663.152 & 66 & 34 & 0 & 0 & 0 \\
\hline 18 & 235.136 & 668.011 & 35 & 65 & 0 & 0 & 0 \\
\hline 19 & 137.636 & 660.919 & 21 & 79 & 0 & 0 & 0 \\
\hline \multirow[t]{2}{*}{$\geq 20$} & 1.714 .311 & 28.000 .028 & 6 & 94 & 0 & 0 & 0 \\
\hline & & & & & 10 & 45 & \\
\hline
\end{tabular}


TABLA 4 (B)

Tabla de Indicadores Escolares (TIE). Total nacional, 1991

\begin{tabular}{|c|c|c|c|c|c|}
\hline$x$ & $S_{(x, x+1)}$ & $\mathrm{xps}_{\mathrm{x}}$ & $q_{x}$ & $\sum_{6}^{\geq 25} S_{x}$ & $e v_{x}$ \\
\hline Edad & Salidas & $\begin{array}{r}\text { Edad ponderada } \\
\text { salidas }\end{array}$ & $\begin{array}{r}\% \text { cociente } \\
\text { de salida }\end{array}$ & Total años & $\begin{array}{r}\text { Esperanza de } \\
\text { vida escolar }\end{array}$ \\
\hline 1 & 8 & $8^{\prime}$ & 9 & 10 & 11 \\
\hline 4 & 0 & 0 & 0 & - & - \\
\hline 5 & 0 & 0 & 0 & - & - \\
\hline 6 & 0 & 0 & 0 & 1173 & 11,5 \\
\hline 7 & 2 & 14 & 2 & 1075 & 10,3 \\
\hline 8 & 0 & 0 & 0 & 975 & 9,4 \\
\hline 9 & 0 & 0 & 0 & 877 & 8,4 \\
\hline 10 & 1 & 10 & 1 & 778 & 7,3 \\
\hline 11 & 1 & 11 & 1 & 678 & 6,3 \\
\hline 12 & 2 & 24 & 2 & 579 & 5,4 \\
\hline 13 & 0 & 0 & 0 & 481 & 4,5 \\
\hline 14 & 8 & 112 & 8 & 385 & 3,5 \\
\hline 15 & 15 & 225 & 17 & 289 & 2,8 \\
\hline 16 & 7 & 112 & 10 & 201 & 2,3 \\
\hline 17 & 31 & 527 & 47 & 128 & 1,4 \\
\hline 18 & 14 & 252 & 40 & 62 & 1,3 \\
\hline 19 & 15 & 285 & 71 & 27 & 0,8 \\
\hline \multirow[t]{3}{*}{$\geq 20$} & 6 & - & 100 & 6 & 0,5 \\
\hline & 96 & 1.572 & & & \\
\hline & $S_{x}-S_{x+1}$ & $\mathrm{~S}_{(\mathrm{x}, \mathrm{x}+1) \mathrm{x}}$ & $\left(\mathrm{S}_{(\mathrm{x}, \mathrm{x}+1)} / \mathrm{S}_{\mathrm{x}}\right) 100$ & $\sum_{6}^{\geq 25} s_{x}$ & $1 / 2+\left(\Sigma\left(S_{x+1}\right) / S_{x}\right)$ \\
\hline${ }_{\bar{x}} e=4,5$ & & & $\bar{x} s=16,4$ & & $e v_{\bar{x}}=11,9$ \\
\hline
\end{tabular}

FUENTES:

Columna 2: MEC, Estadística de Enseñanza en España (E.E.E.), e INE, Censo de Población de 1991. Resultados Nacionales. Columna 3: INE, Censo de Población de 1991. Resultados Nacionales. Columnas 4 a 11: Elaboración propia.

El análisis de las tablas 2, 3 y 4 refleja claramente la evolución de dos décadas. En las edades de enseñanza no obligatoria el incremento de las tasas de escolarización, tanto en las primeras edades (4 y 5 años) como en las de 14 años y más, es importante (Columna 4). Por cada 100 niños de 4 años son 27 más los que entran en el Sistema Educativo en 1981 de lo que lo hacían en 1970, y 25 más en 1991 que en 1981. A la edad de 5 años, en 1970 sólo estaban escolarizados el $69 \%$ de los niños - esto con independencia de que, como señalábamos, parte de los niños de 5 años estuvieran contabilizados en la edad de 6 años-, mientras que en 1991 prácticamente lo están todos. Un país próximo al nuestro, 
Francia, en 1991 tiene todos los niños de 4 y 5 años escolarizados (Viarouge, 1997-98). En España lo está el 95\% de los niños de 4 años y el 99\% de los niños de 5. Y, por otra parte, en las edades de 14 y más años el aumento, especialmente hasta los 17 años, es también notable. En 1970 estaba escolarizada la mitad de la población de 14 años, en 1981 el 84\% y en 1991 el 96 . En cuanto al resto de las edades de enseñanza obligatoria, el incremento de las tasas, dado el alto grado de escolarización en España, se presenta moderado. A la edad de 14 años, obviamente, hay un descenso considerable en la tasa de escolaridad, pues a partir de dicha edad la enseñanza deja de ser obligatoria.

Los indicadores globales de Edad media de entrada $\left({ }_{\bar{x}} e\right)$, Edad media de salida $\left({ }_{\bar{x}} s\right)$ y Esperanza de vida media $\left(\mathrm{ev}_{\overline{\mathrm{x}}}\right)$ nos permiten, de manera sencilla, observar la evolución experimentada en el comportamiento de dichas variables desde 1970 hasta 1991. Lo más relevante a resaltar aquí es el aumento de la Esperanza de vida media, que va de 9,9 años en 1970 a 11,9 años en 1991, debido a la mayor permanencia en el Sistema Educativo de la población estudiante. En 1970 se salía del Sistema Educativo como media a los 14,5 años, mientras que en 1991 se abandona a los 16,4 años.

El indicador más importante, en el estudio de la vida escolar de una población, es la Esperanza de Vida Escolar $\left(e v_{\mathrm{x}}\right)$, Columna 11. Ésta actúa como indicador sintético que resume la experiencia de vida escolar de una generación; por ello, es un indicador único cuando lo que se desea es conocer la evolución del comportamiento de la escolarización a lo largo de un período de tiempo.

En el análisis que estamos haciendo de dos décadas, tomando como referencia los años 1970, 1981 y 1991, observamos en la Esperanza de Vida Escolar un incremento de 1970 a 1981 en 1,7 años, y de 2,3 años de 1970 a 1991. Lo que significa que mientras que en 1970 un individuo a la edad, por ejemplo, de 6 años espera permanecer en el Sistema Educativo 9,2 años más, esto es, hasta tener 15,2 años de edad, en 1991 la expectativa de permanecer en el Sistema Educativos es de 11,5 años, o, lo que es lo mismo, hasta la edad de 17,5 años.

\section{LA ESCOLARIZACIÓN EN LA COMUNIDAD AUTÓNOMA DE LA REGIÓN DE MURCIA}

Como caso particular, veamos ahora el comportamiento de la escolarización en la Región de Murcia para 1991. A nivel regional disponemos de datos completos de estudiantes por edad para el año 1991, gracias a la estadística del censo elaborada por el Centro Regional de Estadística de Murcia (CREM). La fuente de datos utilizada, tanto para la población general como para la población de estudiantes, ha sido la del Censo de Población de 1991 de la Región de Murcia. Resultados Regionales. 
En este caso la serie de edades estudiada ha sido más extensa, desde 4 a 30, ya que, al no pretender hacer comparaciones con las tablas anteriores, nos podemos permitir el aprovechar el mayor desglose de las edades de que disponemos y realizar un estudio más completo.

En la TIE de 1991 de la Región de Murcia (tabla 1) observamos, en los años de Preescolar, unas tasas de escolarización del $90 \%$ a la edad de 4 años y del $98 \%$ a la edad de 5 . En la enseñanza obligatoria la escolarización prácticamente es total, reduciéndose progresivamente a cada edad de manera bastante regular a partir de los 14 años. Señalemos que aunque la ampliación de la enseñanza obligatoria de los 14 a los 16 años, en aplicación de la Ley Orgánica de Ordenación General del Sistema Educativo (LOGSE), se inició en 1990, su puesta en escena, sin embargo, se ha ido dosificando a lo largo de la década. Culmina su generalización en el curso académico 1999-2000, lográndose, con el cambio de milenio, la escolarización obligatoria para todos los españoles de 6 a 16 años.

En relación a la obligatoriedad de la enseñanza en España, conviene recordar que la primera vez que se fija por ley dicha obligatoriedad fue en 1857, comprendida entre los 6 y los 9 años de edad, aunque en realidad existieron muchas dificultades que impidieron que muchos niños recibieran instrucción en esa época, porque aunque es la obligatoriedad un precepto en sus leyes, la falta de medidas para llevarla a cabo hizo que no fuese del todo real (Turín, 1967). Es hacia 1880 cuando se plantea la necesidad de ampliar la edad escolar obligatoria tanto por debajo de los 6 años como por encima de los 9, con el fin de que la enseñanza incluyera el alto porcentaje del alumnado que recibía instrucción sin estar dentro de los límites que señalaba la ley. En 1885 el alumnado en edad escolar se igualaba al alumnado fuera de la obligatoriedad —-menor de 6 y mayor de 9 años- (Ramos, 1987).

Siguiendo con el análisis de la TIE de la Región de Murcia, conviene señalar que a los 18 años la mitad de la población está todavía dentro del Sistema Educativo.

En 1991 la Edad media de entrada $\left({ }_{\bar{x}}\right.$ e) en la Región de Murcia es de 4,3 años, la Edad media de salida $(\bar{x} s)$ de 18,6 años, y la Esperanza de vida media $\left(e v_{\bar{x}}\right)$ de 14,3 años.

La escolarización por género ofrece pequeñas diferencias a nivel global (tablas 4 y 5). El indicador medio de entrada difiere en 1,2 meses a favor de la mujer, en el sentido de que entra como media 1,2 meses antes a formar parte del Sistema Educativo. Y, por otra parte, las mujeres salen más tarde; lo hacen concretamente 4,8 meses después que los hombres, con lo que su permanencia en el Sistema Educativo viene a ser de 6 meses más.

Si observamos el indicador Esperanza de Vida Escolar $\left(\mathrm{ev}_{\mathrm{x}}\right)$, tabla 1, advertimos que a la edad de 6 años un individuo espera permanecer en el Sistema Educativo 13,3 años; espera llegar, como media, hasta la edad de 19,3 años. Las mujeres, 0,4 meses más que los hombres (tablas 5 y 6 ). 
TABLA 5 (A)

Tabla de Indicadores Escolares (TIE). Región de Murcia, 1991. Mujeres

\begin{tabular}{|c|c|c|c|c|c|c|c|}
\hline$x$ & $\mathrm{E}_{\mathrm{x}}$ & $P_{x}$ & $S_{x}$ & $N\left(S_{x}\right)$ & $e_{(x, x+1)}$ & $\mathrm{xpe}_{\mathrm{x}}$ & $\mathrm{p}_{\mathrm{x}}$ \\
\hline Edad & Estudiantes & $\begin{array}{r}\text { Población } \\
\text { total }\end{array}$ & $\begin{array}{c}\text { \% tasa } \\
\text { escola- } \\
\text { rización }\end{array}$ & $\begin{array}{r}\% \text { tasa } \\
\text { no esco- } \\
\text { larizados }\end{array}$ & Entradas & $\begin{array}{r}\text { Edad } \\
\text { ponderada } \\
\text { entradas }\end{array}$ & $\begin{array}{r}\% \text { cociente } \\
\text { de } \\
\text { entrada }\end{array}$ \\
\hline 1 & 2 & 3 & 4 & 5 & 6 & $6^{\prime}$ & 7 \\
\hline 4 & 5.886 & 6.571 & 90 & 10 & 8 & 32 & 80 \\
\hline 5 & 6.648 & 6.812 & 98 & 2 & 1 & 5 & 50 \\
\hline 6 & 6.883 & 6.949 & 99 & 1 & 1 & 6 & 100 \\
\hline 7 & 7.243 & 7.276 & 100 & 0 & 0 & 0 & 0 \\
\hline 8 & 7.812 & 7.839 & 100 & 0 & 0 & 0 & 0 \\
\hline 9 & 8.198 & 8.223 & 100 & 0 & 0 & 0 & 0 \\
\hline 10 & 8.618 & 8.639 & 100 & 0 & 0 & 0 & 0 \\
\hline 11 & 8.883 & 8.932 & 99 & 1 & 0 & 0 & 0 \\
\hline 12 & 9.296 & 9.376 & 99 & 1 & 0 & 0 & 0 \\
\hline 13 & 8.884 & 9.041 & 98 & 2 & 0 & 0 & 0 \\
\hline 14 & 8.797 & 9.427 & 93 & 7 & 0 & 0 & 0 \\
\hline 15 & 7.956 & 9.577 & 83 & 17 & 0 & 0 & 0 \\
\hline 16 & 6.979 & 9.591 & 73 & 27 & 0 & 0 & 0 \\
\hline 17 & 6.042 & 9.436 & 64 & 36 & 0 & 0 & 0 \\
\hline 18 & 5.242 & 9.358 & 56 & 44 & 0 & 0 & 0 \\
\hline 19 & 4.269 & 9.086 & 47 & 53 & 0 & 0 & 0 \\
\hline 20 & 3.617 & 9.263 & 39 & 61 & 0 & 0 & 0 \\
\hline 21 & 2.977 & 9.096 & 33 & 67 & 0 & 0 & 0 \\
\hline 22 & 2.436 & 9.081 & 27 & 73 & 0 & 0 & 0 \\
\hline 23 & 1.977 & 9.264 & 21 & 79 & 0 & 0 & 0 \\
\hline 24 & 1.521 & 9.128 & 17 & 83 & 0 & 0 & 0 \\
\hline 25 & 1.084 & 8.852 & 12 & 88 & 0 & 0 & 0 \\
\hline 26 & 874 & 8.772 & 10 & 90 & 0 & 0 & 0 \\
\hline 27 & 627 & 8.652 & 7 & 93 & 0 & 0 & 0 \\
\hline 28 & 568 & 7.996 & 7 & 93 & 0 & 0 & 0 \\
\hline 29 & 415 & 7.794 & 5 & 95 & 0 & 0 & 0 \\
\hline \multirow[t]{2}{*}{$\geq 30$} & 3.412 & 280.980 & 1 & 99 & 0 & 0 & 0 \\
\hline & & & & & 10 & 43 & \\
\hline
\end{tabular}


TABLA 5 (B)

Tabla de Indicadores Escolares (TIE). Región de Murcia, 1991. Mujeres

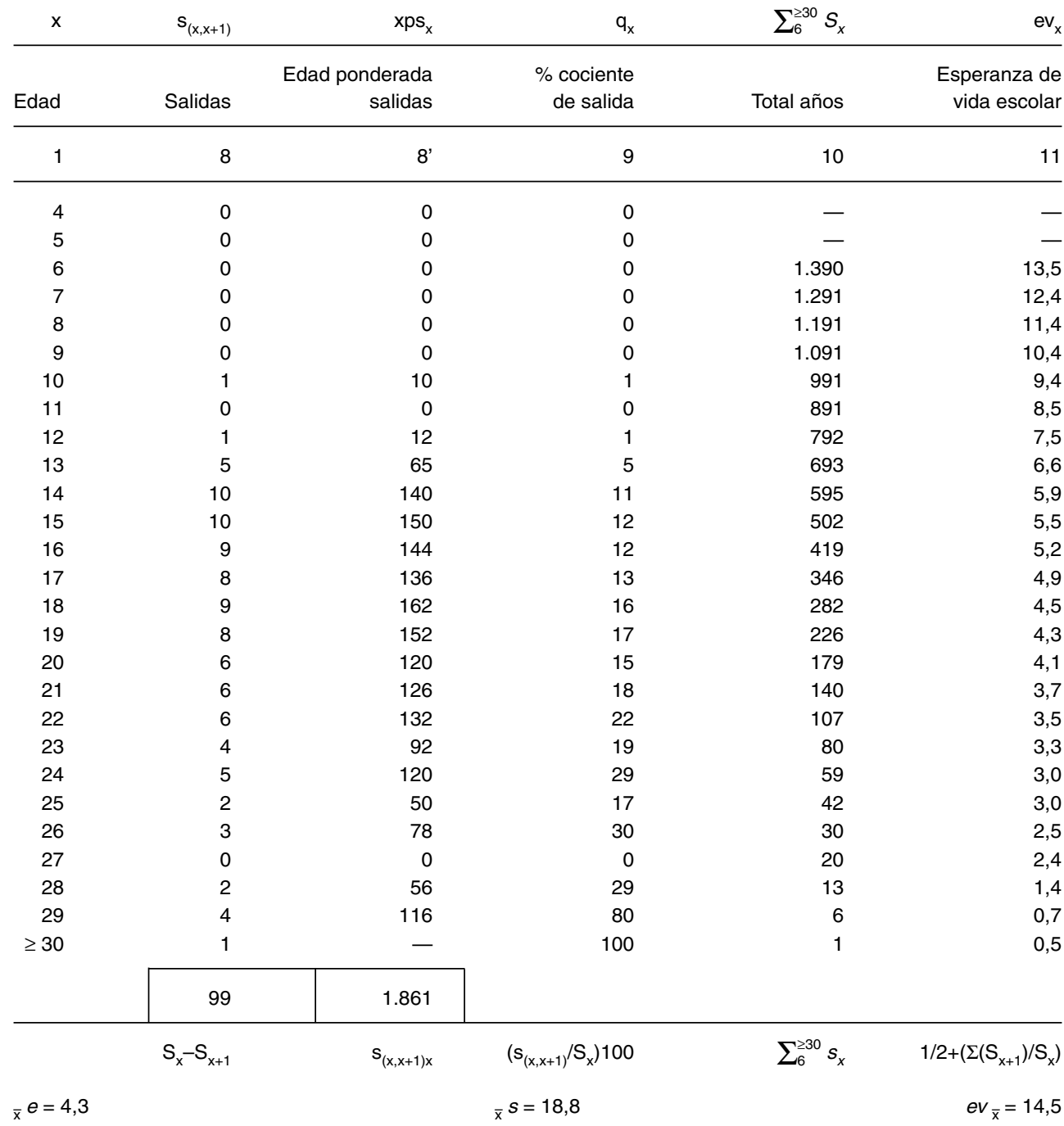

Fuentes:

Columnas 2 y 3: Centro Regional de Estadística de Murcia, Censo de Población de 1991 de la Región de Murcia. Resultados Regionales. Columnas 4 a 11: Elaboración propia. 
TABLA 6 (A)

Tabla de Indicadores Escolares (TIE). Región de Murcia, 1991. Hombres

\begin{tabular}{|c|c|c|c|c|c|c|c|}
\hline$x$ & $\mathrm{E}_{\mathrm{x}}$ & $P_{x}$ & $S_{x}$ & $N\left(S_{x}\right)$ & $e_{(x, x+1)}$ & $\mathrm{xpe}_{\mathrm{x}}$ & $\mathrm{p}_{\mathrm{x}}$ \\
\hline Edad & Estudiantes & $\begin{array}{r}\text { Población } \\
\text { total }\end{array}$ & $\begin{array}{c}\text { \% tasa } \\
\text { escola- } \\
\text { rización }\end{array}$ & $\begin{array}{r}\% \text { tasa } \\
\text { no esco- } \\
\text { larizados }\end{array}$ & Entradas & $\begin{array}{r}\text { Edad } \\
\text { ponderada } \\
\text { entradas }\end{array}$ & $\begin{array}{r}\% \text { cociente } \\
\text { de } \\
\text { entrada }\end{array}$ \\
\hline 1 & 2 & 3 & 4 & 5 & 6 & $6^{\prime}$ & 7 \\
\hline 4 & 6.262 & 6.990 & 90 & 10 & 7 & 28 & 70 \\
\hline 5 & 7.079 & 7.266 & 97 & 3 & 2 & 10 & 67 \\
\hline 6 & 7.264 & 7.313 & 99 & 1 & 1 & 6 & 100 \\
\hline 7 & 7.711 & 7.747 & 100 & 0 & 0 & 0 & 0 \\
\hline 8 & 8.188 & 8.215 & 100 & 0 & 0 & 0 & 0 \\
\hline 9 & 8.602 & 8.622 & 100 & 0 & 0 & 0 & 0 \\
\hline 10 & 9.265 & 9.300 & 100 & 0 & 0 & 0 & 0 \\
\hline 11 & 9.566 & 9.623 & 99 & 1 & 0 & 0 & 0 \\
\hline 12 & 9.765 & 9.840 & 99 & 1 & 0 & 0 & 0 \\
\hline 13 & 9.634 & 9.739 & 99 & 1 & 0 & 0 & 0 \\
\hline 14 & 9.418 & 9.985 & 94 & 6 & 0 & 0 & 0 \\
\hline 15 & 8.271 & 10.026 & 82 & 18 & 0 & 0 & 0 \\
\hline 16 & 6.565 & 9.955 & 66 & 34 & 0 & 0 & 0 \\
\hline 17 & 5.687 & 10.017 & 57 & 43 & 0 & 0 & 0 \\
\hline 18 & 4.564 & 9.593 & 48 & 52 & 0 & 0 & 0 \\
\hline 19 & 3.763 & 9.498 & 40 & 60 & 0 & 0 & 0 \\
\hline 20 & 3.277 & 9.636 & 34 & 66 & 0 & 0 & 0 \\
\hline 21 & 2.759 & 9.520 & 29 & 71 & 0 & 0 & 0 \\
\hline 22 & 2.363 & 9.577 & 25 & 75 & 0 & 0 & 0 \\
\hline 23 & 1.893 & 9.612 & 20 & 80 & 0 & 0 & 0 \\
\hline 24 & 1.488 & 9.280 & 12 & 84 & 0 & 0 & 0 \\
\hline 25 & 1.136 & 9.144 & 12 & 88 & 0 & 0 & 0 \\
\hline 26 & 838 & 9.048 & 9 & 91 & 0 & 0 & 0 \\
\hline 27 & 641 & 8.748 & 7 & 93 & 0 & 0 & 0 \\
\hline 28 & 450 & 7.995 & 6 & 94 & 0 & 0 & 0 \\
\hline 29 & 381 & 7.714 & 5 & 95 & 0 & 0 & 0 \\
\hline \multirow[t]{2}{*}{$\geq 30$} & 2.588 & 252.660 & 1 & 99 & 0 & 0 & 0 \\
\hline & & & & & 10 & 44 & \\
\hline
\end{tabular}


TABLA $6(B)$

Tabla de Indicadores Escolares (TIE). Región de Murcia, 1991. Hombres

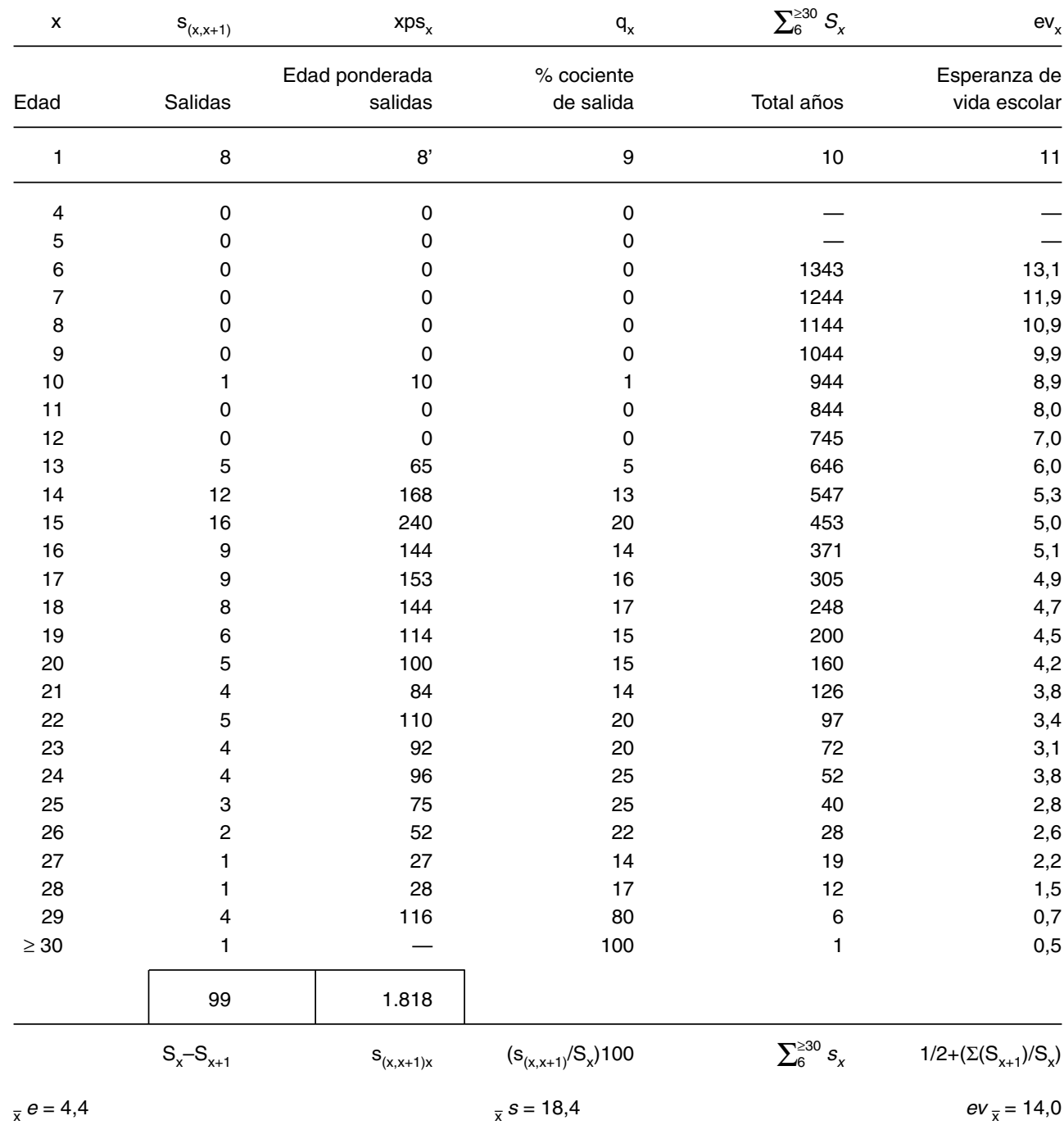

Fuentes:

Columnas 2 y 3: Centro Regional de Estadística de Murcia, Censo de Población de 1991 de la Región de Murcia. Resultados Regionales. Columnas 4 a 11: Elaboración propia. 
Otras diferencias por género las encontramos cuando comparamos las tasas de escolarización edad a edad a partir de los 16 años. Concretamente en el intervalo de edad de 16 a 21 años, las mujeres superan a los hombres en un valor medio, para cada una de esas edades, de 6 puntos, como puede observarse de manera gráfica en la Pirámide de Tasas de escolarización (figura 1).

FIGURA 1

Tasas de escolarización por sexo

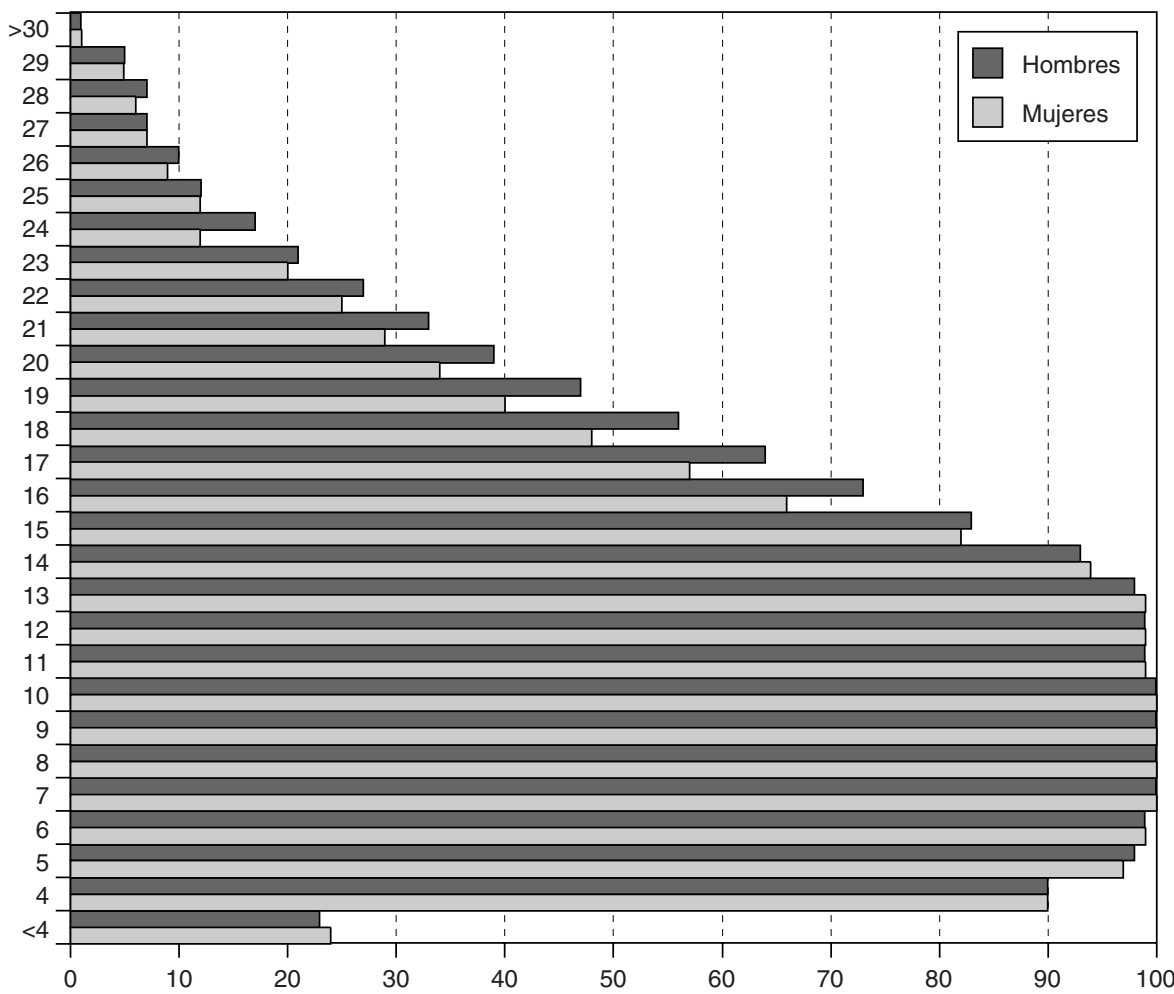

FUENTE:

Centro Regional de Estadística de Murcia, Censo de Población de 1991 de la Región de Murcia. Resultados Regionales. Elaboración propia. 


\section{CONCLUSIONES}

Del conjunto de observaciones realizadas en el artículo en relación a la Demografía de la Educación, queremos destacar, a modo de conclusiones, las siguientes:

- Este campo científico, disciplinalmente hablando, es Demografía. Por tanto, éste debe proveer a la Educación de todo su bagaje conceptual e instrumental.

- En este territorio científico se analizan una serie de comportamientos, al igual que lo hace la Demografía, ya que dentro del Sistema Educativo se dan también procesos que determinan la formación (entradas en el Sistema Educativo), la conservación (permanencia en el Sistema Educativo) y desaparición (salidas del sistema Educativo) de la población.

- Frente a las abundantes y potentes técnicas de análisis que ha producido la Demografía, contrasta el escaso desarrollo de las mismas en Demografía de la Educación, lo que explica el déficit de análisis existente en este campo.

- La técnica de análisis que proponemos para examinar los comportamientos de la escolarización de la población (Tabla de Indicadores Escolares) permite, por una parte, tener una visión global de todo el proceso que experimenta la escolarización para un horizonte temporal determinado; pero, por otra, también facilita el análisis de cada uno de los elementos que conforman dicho proceso, bien sean las entradas, como la permanencia o las salidas del Sistema Educativo.

- La construcción de la Tabla de Indicadores Escolares precisa de buena información estadística. Los datos deben permitir, por una parte, construir series temporales que faciliten el análisis de los procesos de escolarización de la población y, por otra, deben estar lo suficientemente desagregados como para poder cumplimentar los requerimientos de la Tabla; sólo si es así, ésta será, realmente, un instrumento eficaz en el examen de los comportamientos de la escolarización que tienen lugar al interior del Sistema Educativo. 


\section{BIBLIOGRAFÍA}

CENTRO REGIONAL DE ESTAdístiCA DE MURCIA (1994): Censo de Población de 1991 de la Región de Murcia. Resultados Regionales, tomo I, Región de Murcia, Consejería de Fomento y Trabajo, Murcia.

CLERC, P. (1977): «Demografía escolar», en M. Debesse y G. Mialaret, Aspectos sociales de la educación-II, Oikostau, S.A. Ediciones, Barcelona.

DEPARTAMENTO DE EDUCACIÓN, UNIVERSIDADES E INVESTIGACIÓN. GOBIERNO VASCO (1990): Demografía y Educación, Servicio Central de Publicaciones, Bilbao.

FERNÁNDEZ, N. G. (1987): Escolarización y sociedad en Galicia (1875-1900), Imprenta Universitaria, Santiago de Compostela.

FURET, F. (1978): «Lo cuantitativo en historia», en Jacques Le Goff y Pierre Nora (eds.), Hacer la Historia, vol. 1, Laia, Barcelona.

GUEREÑA, J.-L., y VIÑAO, A. (1996): Estadística escolar, proceso de escolarización y sistema educativo nacional en España (1750-1850), EUB, S.L., Barcelona.

GUYOT, J. L. (1995): Vieilles marmites et meilleures soupes ou quand le démographe se doit de faire mentir l'adage populaire, Institut de Démographie de I'U.C.L., Louvain-la-Neuve.

- (1996): Méthodes pour l'analyse des sous-populations scolaires. Accès et accessibilité à un système d'enseignement donné, «Working Paper», n. ${ }^{\circ} 181$, Institut de Démographie de l'U.C.L., Louvain-la-Neuve.

INSTITUTO NACIONAL DE ESTADÍSTICA (1972): Estadística de la Enseñanza en España, Curso 1970-71, Madrid.

- (1974): Censo de la Población de España según la inscripción realizada el 31 de diciembre de 1970. Total Nacional, tomo III, Características de la Población, Madrid.

- (1985): Censo de Población de 1981. Resultados Nacionales, tomo I, vol. I, Características de la Población, Madrid.

- (1985): Estadística de la Enseñanza en España, Curso 1981-82, Madrid.

- (1988): Estadística de la Enseñanza en España, Curso 1984-85, Madrid.

- (1994): Censo de Población de 1991. Resultados Nacionales, tomo I, Características Generales de la Población, Madrid.

LEGUINA, J. (1989): Fundamentos de demografía, Siglo Veintiuno de España Editores, S.A., Madrid.

LIVI-BACCI, M. (1993): Introducción a la demografía, Editorial Ariel, S.A., Barcelona.

LOZANO, C. (1990): La escolarización. Historia de la enseñanza, Montesinos Editor, S.A., Barcelona.

MINISTERIO DE EDUCACIÓNY CIENCIA (1994): Estadística de la Enseñanza en España. 1991/92. Niveles de Preescolar, Infantil, General Básica y EE. Medias, Madrid.

ROSEAU, M. (1998): La démographie scolaire en France de 1990 a 1998, MST Sciences Sociales Appliquées, París.

RUIZ, R. (1986): Historia de la Educación en Zamora. Escolarización y sociedad en la provincia de Zamora en la segunda mitad del siglo XIX, tomo 2, Instituto de Estudios Zamoranos «Florián de Ocampo».

TORRES MORA, J. A. (1991): «Demografía educativa de los años ochenta: el nacimiento de una meritocracia bastarda», en Educación y Sociedad, n. ${ }^{\circ}$ 8, Madrid, pp. 25-58.

VIAROUGE, N. (1997): Recherche Bibliographique sur la Démographie Scolaire en France (1990-1998), MST Sciences Sociales Appliquées, París. 


\section{ABSTRACT}

In this article we propose, taking as reference points the theoretical and instrumental experience of demography, to equip the demography of education with valid instruments for analysis, so as to create its own body of knowledge which hitherto has scarcely been developed.

With this objective in mind, an attempt is made to delve into the analysis of some aspects of schooling using a series of measurement techniques that reveal rather remarkable features of school population. Specifically, a base instrument is developed and we have called this the Table of Scholastic Indicators, which is presented as a powerful measurement instrument, capable of furnishing the information necessary for responding to the basic objective of this article. 